TAIWANESE JOURNAL OF MATHEMATICS

Vol. 11, No. 1, pp. 51-62, March 2007

This paper is available online at http://www.math.nthu.edu.tw/tjm/

\title{
JORDAN DERIVATIONS OF SOME CLASSES OF MATRIX RINGS
}

\author{
Nader M. Ghosseiri
}

\begin{abstract}
Let $R$ be a 2-torsionfree ring with identity and $S$ be a subring of the ring $M_{n}(R)$ that contains the ring $T_{n}(R)$ of all upper triangular matrices over $R$; that is, $T_{n}(R) \subseteq S \subseteq M_{n}(R)$. The goal of this paper is to describe a Jordan derivation $\Delta$ on $S$. The main result states that $\Delta$ can be uniquely represented as the sum of a derivation and a very special Jordan derivation. This result describes also the structure of every derivation on the ring $S$ which is an extension of a result of S.P. Coelho and C.P. Milies and a result of S. Jøndrup. Moreover, one of the corollaries of the main theorem covers the classical result by Jacobson and Rickart stating that there are no proper Jordan derivations on $M_{n}(R)$, and a more recent result by D. Benkovic that there are no proper Jordan derivations on the algebra $T_{n}(R)$.
\end{abstract}

\section{INTRODUCTION}

Let $R$ be a ring. An additive mapping $d: R \rightarrow R$ is said to be a derivation if for all $a, b \in R, d(a b)=d(a) b+a d(b)$. For each $a \in R$, the mapping $d_{a}: R \rightarrow R$ given by $d_{a}(x)=a x-x a, x \in R$, is easily seen to be a derivation on $R$. $d_{a}$ is called the inner derivation induced by $a$. The additive mapping $\delta: R \rightarrow R$ defined by $\delta(a b)=\delta(b) a+b \delta(a)$ for all $a, b \in R$ is called an antiderivation. The additive mapping $\Delta: R \rightarrow R$ defined by $\Delta(a b+b a)=\Delta(a) b+a \Delta(b)+\Delta(b) a+$ $b \Delta(a)$ for all $a, b \in R$ is called a Jordan derivation. Obviously, each derivation or antiderivation is a Jordan derivation, but the converses are not true (see examples 1-4 bellow).

Let $n$ be a positive integer. By $M_{n}(R)$ we denote the ring of all $n \times n$ marices over $R$, by $T_{n}(R)$ we denote the ring of all $n \times n$ upper triangular matrices over $R$, and $e_{i j}$ stands for the usual matrix unit. Let $R$ be a 2-torsionfree ring with identity, $n \geq 2$, and $S$ be a subring of $M_{n}(R)$ that contains $T_{n}(R)$; i.e. $T_{n}(R) \subseteq S \subseteq$

Received November 29, 2004, revised April 16, 2005.

Communicated by Shun-Jen Cheng.

2000 Mathematics Subject Classification: 16W25.

Key words and phrases: Derivation, Antiderivation, Jordan derivation, Matrix ring. 
$M_{n}(R)$. In this paper we determine the structure of Jordan derivations on $S$. This result generalizes some known results, and there are some analogies between this result and some others.

In 1993 S.P. Coelho and C.P. Milies [2] proved that if $R$ is a ring with identity and $d$ is a derivation on $T_{n}(R)$, then there exists a derivation $\delta: R \rightarrow R$ and a matrix $A \in T_{n}(R)$ such that $d=\bar{\delta}+d_{A}$, where $\bar{\delta}\left(\left[x_{i j}\right]\right)=\left[\delta\left(x_{i j}\right)\right]$ for each $\left[x_{i j}\right] \in T_{n}(R)$ and $d_{A}$ is the inner derivation induced by $A$. In $1995 \mathrm{~S}$. Jødrup [4] determined a similar structure for $C$-derivations on $M_{n}(A)$ and $T_{n}(A)$, where $A$ is a $C$-algebra. In Corollary 1 we extend these results to any ring $S$ such that $T_{n}(R) \subseteq S \subseteq M_{n}(R)$. By a classical result of Jacobson and Rickart the ring $M_{n}(R)$ has no proper Jordan derivations [3, Theorem 22]; more recently, in 2005 D. Benkovic [1] has shown that if $R$ is a 2-torsionfree commutative ring with identity and $n \geq 2$, then $T_{n}(R)$ has no proper $R$-linear Jordan derivations. Corollary 2 establishes these results at once. In [1, Theorem 1.1] it has also been proved that if $R$ is a commutative 2torsionfree ring with identity, then every $R$-linear Jordan derivation from $T_{n}(R)$ into a $T_{n}(R)$-bimodule $M$ is the sum of a derivation and an antiderivation. For the case that $M=T_{n}(R)$ and $n \geq 3$ this result is an special case of Theorem 1. For more studies concerning Jordan derivations we refer the reader to [1] and the references therein.

In section 2 we shall consider singular Jordan derivations on $S$ and in particular observe the connections between them and antiderivations. In section 3 we will state our main results. In particular, Theorem 1 states that every Jordan derivation $\Delta: S \rightarrow S$ can be uniquely represented as the sum of a derivation $d: S \rightarrow S$ and a singular Jordan derivation $\delta: S \rightarrow S$, and in Corollary 1 we will describe the structure of derivations on $S$. Section 4 is devoted to the proof of Theorem 1.

\section{Singular Jordan Derivations}

The purpose of this section is to introduce the concept of a singular Jordan derivation, and gather together some useful observations about this concept.

We begin by the following important observations which can be easily verified.

Remark 1. The following two conditions are equivalent:

(i) $S$ is a subring of $M_{n}(R)$ such that $T_{n}(R) \subseteq S$.

(i) There exist ideals $I_{i j}, 1 \leq i, j \leq n$, of $R$ such that $I_{i j}=R$ for all $i \leq$ $j, I_{i j} I_{j k} \subseteq I_{i k}$ for all $i, j, k$, and

$$
S=\left\{\Sigma_{i, j} x_{i j} e_{i j}: x_{i j} \in I_{i j}\right\} .
$$

The notion of a singular Jordan derivation naturally appears when studying Jordan derivations on $S$. To see this we consider two cases: 
First, let $n=2$ and let $S$ be a ring such that $T_{n}(R) \subseteq S \subseteq M_{n}(R)$. Then by Remark 1 , there exists an ideal $I$ in $R$ such that

$$
S=\left(\begin{array}{cc}
R & R \\
I & R
\end{array}\right)
$$

We have

Remark 2. Let $f: I \rightarrow R$ be an additive map satisfying

(i) $f(x y)=y f(x)$ for all $x \in I, y \in R$;

(ii) $f(y x)=f(x) y$ for all $x \in I, y \in R$;

(iii) $f\left(x^{2}\right)=0$ for all $x \in I$.

Then the mapping $\delta: S \rightarrow S$ defined by

$$
\delta\left(x e_{21}\right)=f(x) e_{12} \text { and } \delta\left(x e_{i j}\right)=0 \text { for all } e_{i j} \neq e_{12}
$$

is a Jordan derivation.

One can easily check that $\delta$ is a Jordan derivation, but not a derivation if $f \neq 0$. Such a Jordan derivation will be called a singular Jordan derivation. Also, it is not hard to see that $\delta$ is an antiderivation if and only if $f\left(I^{2}\right)=0$. The next two examples illustrate these instances.

Example 1. Let $R$ be a commutative ring with identity and $I$ be a nonzero ideal of $R$ such that $I^{2}=0$. The mapping $f: I \rightarrow R$ defined by $f(x)=x, x \in I$ satisfies all conditions of Remark 2; moreover, $f\left(I^{2}\right)=0$. Therefore, the map $\delta: S \rightarrow S$, where

$$
S=\left(\begin{array}{cc}
R & R \\
I & R
\end{array}\right)
$$

is a singular Jordan derivation and at the same time an antiderivation which is not a derivation.

Example 2. Let $R[x, y]$ be the polynomial ring in $x, y$ over $R$, where $R$ is again a commutative ring with identity. Let $I$ be its ideal generated by $x^{2}, y^{2}$, and $2 x y$ and set

$$
R^{\prime}=R[x, y] / I .
$$

Assume that $I^{\prime}$ is the ideal of $R^{\prime}$ generated by $x+I, y+I$, and put

$$
S=\left(\begin{array}{cc}
R^{\prime} & R^{\prime} \\
I^{\prime} & R^{\prime}
\end{array}\right) .
$$


One can easily verify that $f: I^{\prime} \rightarrow R^{\prime}$ defined by $f\left(x^{\prime}\right)=x^{\prime}$ for every $x^{\prime} \in I^{\prime}$, satisfies all conditions of Remark 2. Therefore, $\delta: S \rightarrow S$ is a Jordan derivation that is not a derivation; that is, $\delta$ is a singular Jordan derivation. Since $f((x+$ $I)(y+I))=x y+I \neq 0$, we have $f\left(I^{\prime 2}\right) \neq 0$, whence $\delta$ is not an antiderivation.

Next, assume that $n \geq 3$ and let $S$ be a ring such that $T_{n}(R) \subseteq S \subseteq M_{n}(R)$. By $I_{i j}$ we denote the appropriate ideals of $R$ illustrated in Remark 1. We have

Remark 3. If an additive map $f: I_{n 1} \rightarrow R$ satisfies

(i) $f(x y)=y f(x)$ for all $x \in I_{n 1}, y \in R$;

(ii) $f(y x)=f(x) y$ for all $x \in I_{n 1}, y \in R$;

(iii) $f\left(I_{n j} I_{n 1}\right)=f\left(I_{n 1} I_{j 1}\right)=f\left(I_{n j} I_{j 1}\right)=0$ for all $1<j<n$,

then $\delta: S \rightarrow S$ defined by

$$
\delta\left(x e_{n 1}\right)=f(x) e_{1 n} \text { and } \delta\left(x e_{i j}\right)=0 \text { for all } e_{i j} \neq e_{n 1}
$$

is a Jordan derivation.

In fact, from $\left(x e_{n 1}\right) e_{12}=x e_{n 2}$ it follows that $I_{n 1} \subseteq I_{n 2}$, whence $I_{n 1}{ }^{2} \subseteq I_{n 1} I_{n 2}$, so that by (iii), $f\left(I_{n 1}{ }^{2}\right) \subseteq f\left(I_{n 1} I_{n 2}\right)=0$. Now it is easy to see that $\delta$ is an antiderivation and therefore a Jordan derivation, but not a derivation if $f \neq 0$. For $n \geq 3$, such a map $\delta$ will be called a singular Jordan derivation. Here is an example:

Example 3. Let $n \geq 3, R$ be a commutative ring with identity, and $I$ be a nonzero ideal of $R$ such that $I^{2}=0$. Let the ring $S$ be such that $I_{i j}=I$ for all $i>j$ and $I_{i j}=R$ for all $i \leq j$. Then the embedding $f: I \rightarrow R$ satisfies all conditions of Remark 3. Accordingly $\delta: S \rightarrow S$ is an antiderivation, but not a derivation.

This section ends with some situations in which $S$ has no nonzero singular Jordan derivations:

Remark 4. If the ideal $I_{n 1}$ of $R$ is trivial; i.e. $I_{n 1}=0$ or $I_{n 1}=R$, then $S$ does not have nonzero singular Jordan derivations. In particular, this holds true for $T_{n}(R)$ or $S_{n}(R)$.

Proof. The case $I_{n 1}=0$ is obvious. Let $I_{n 1}=R$. Then $f\left(x^{2}\right)=0$ for all $x \in R$, and so $f(1)=0$. Therefore, each of Remark 2(i) or Remark 3(i) implies that

$$
f(y)=f(1 y)=y f(1)=0
$$


for all $y \in R$. Therefore, $S$ does not have nonzero singular Jordan derivations.

Remark 5. Let $R$ be a prime ring and $S$ be a ring such that $T_{n}(R) \subseteq S \subseteq$ $M_{n}(R)$. Then:

(i) If $n \geq 3$, then $S$ does not have nonzero singular Jordan derivations.

(ii) If $n=2$ and $\operatorname{char}(R) \neq 2$, then $S$ does not have nonzero singular Jordan derivations.

Proof. The case $I_{n 1}=0$ is obvious. Suppose therefore that $I_{n 1} \neq 0$.

Let $n \geq 3$. Since $f\left(I_{n 1}{ }^{2}\right)=0$, Remark 3(i) yields

$$
0=f(x(y r))=\operatorname{yr} f(x)
$$

for all $x, y \in I_{n 1}, r \in R$. Since $R$ is prime, we conclude that $f=0$, whence nonzero singular Jordan derivations do not exist on $S$.

Assume $n=2$. Then for every $x \in I_{21}$ we have $f\left(x^{2}\right)=x f(x)=0$. Linearizing we get $x f(y)=y f(x)=0$ for all $x, y \in I_{21}$. Replacing $y$ by $y r$, where $r \in R$, we get $\operatorname{xr} f(y)+\operatorname{yr} f(x)=0$ which implies that

$$
2 x R f(x)=0,
$$

and this implies the desired conclusion.

From $f\left(I_{n 1}{ }^{2}\right)=0$ and both (i) and (ii) in Remark 3 it follows that $f(x y)=$ $y f(x)=f(y) x=0$ for all $x, y \in I_{n 1}$. Thus:

Remark 5. Let $n \geq 3$ and $S$ be a ring such that $T_{n}(R) \subseteq S \subseteq M_{n}(R)$. If either $\operatorname{ann}_{l}\left(I_{n 1}\right) \cap \operatorname{ann}_{r}\left(I_{n 1}\right)=0$ or $I_{n 1}{ }^{2}=I_{n 1}$, then $S$ does not have nonzero singular Jordan derivations.

\section{MAin Results}

Throughout this section, $R$ will be a 2 -torsionfree ring with identity, $n \geq 2$, and $S$ will be a subring of $M_{n}(R)$ that contains $T_{n}(R)$.

Theorem 1. Let $\Delta: S \rightarrow S$ be a Jordan derivation. Then there exists a unique derivation $d: S \rightarrow S$ and a unique singular Jordan derivation $\delta: S \rightarrow S$ such that $\Delta=d+\delta$. Moreover, $d$ is the sum of an inner derivation $d_{A}$ and $a$ derivation $d_{R}$ arising from a derivation on $R$, and $\delta$ is an antiderivation if $n \geq 3$. 
Since the last assertion is interesting in its own right, let us record it as a separate statement. As we shall see in the proof we do not use the assumption that $R$ is 2-torsionfree.

Corollary 1. Let $d: S \rightarrow S$ be a derivation. Then there is an inner derivation $d_{A}: S \rightarrow S$ and a derivation $d_{R}: S \rightarrow S$ arising from a derivation on $R$ such that $d=d_{A}+d_{R}$. If $d=d_{A^{\prime}}+d_{R}^{\prime}$, for some matrix $A^{\prime}$ and a derivation $d_{R}^{\prime}$ arising from a derivation on $R$, then there exists $r \in R$ such that $d_{A^{\prime}}=d_{A}+d_{r I}$.

From Theorem 1 and Remarks 4 and 5 from the preceding section we have immediately the following corollaries.

Corollary 2. Every Jordan derivation on any of the rings $M_{n}(R)$ and $T_{n}(R)$ is a derivation.

Corollary 3. If $R$ is a prime ring, then every Jordan derivation on $S$ is a derivation.

We end this section by an example. This shows that if we have no torsion assumption on $R$, then (a) the Jordan derivation in Theorem 1 may not have the structure described there; (b) the Jordan derivations in Corollary 2 and Corollary 3 (for the case $n=2$ ) may fail to be derivations on $S$.

Example 4. Let $U$ be the ring of integers modulo 2, $R$ be the ring $U[x]$, and $S$ be either of $M_{2}(R)$ or $T_{2}(R)$. For each $P=\left[f_{i j}\right] \in S$ define $\Delta(P)=\left(f_{11}{ }^{\prime}+f_{22}{ }^{\prime}\right) I$, where $I$ is the identity martix and $f_{i i}{ }^{\prime}$ is the usual derivation of $f_{i i}$. Then it is easily verified that the Jordan derivation $\Delta$ is not of the form described by Theorem 1 . Also, $\Delta$ is neither a derivation nor an antiderivation.

\section{Proof of the Main Theorem}

The following observations hold true in any 2-torsionfree ring $S$. If $\Delta: S \rightarrow S$ is a Jordan derivation, then:

$$
\begin{gathered}
\Delta\left(a^{2}\right)=\Delta(a) a+a \Delta(a) \\
\Delta(a b a)=\Delta(a) b a+a \Delta(b) a+a b \Delta(a) \\
\text { (2) } \Delta(a b c+c b a)=\Delta(a) b c+a \Delta(b) c+a b \Delta(c)+\Delta(c) b a+c \Delta(b) a+c b \Delta(a)
\end{gathered}
$$

for all $a, b, c \in S$. Indeed, (1) follows from the definition and the assumption that $S$ is 2-torsionfree; applying $\Delta$ to the identity $2 a b a=a(a b+b a)+(a b+b a) a-$ 
$\left(a^{2} b+b a^{2}\right)$ we get (2), and linearizing (2) we obtain (3).

Theorem 1 will be proved in three steps. In the first step we shall find an appropriate singular Jordan derivation arising from the Jordan derivation $\Delta$; in the second step we will find $A \in S$ that gives rise to an inner derivation $d_{A}$, and in the last step we will prove that the remaining part is a derivation arising from a derivation on $R$.

Step 1. Let $\Delta: S \rightarrow S$ be a Jordan derivation. Define $\delta: S \rightarrow S$ by

$$
\begin{gathered}
\delta\left(x e_{i j}\right)=0 \text { for all } e_{i j} \neq e_{n 1}, \text { and } \\
\delta\left(x e_{n 1}\right)=e_{11} \Delta\left(x e_{n 1}\right) e_{n n}=f(x) e_{1 n},
\end{gathered}
$$

where the mapping $f: I_{n 1} \rightarrow R$ is determined by $\delta$ and for each $x \in I_{n 1}, f(x)$ is the entry of the matrix $\Delta\left(x e_{n 1}\right)$ at the position $(1, n)$. Since $\delta$ is an additive map, so is $f$.

Lemma 1. $\delta$ is a singular Jordan derivation.

Proof. It suffices to show that the map $f: I_{n 1} \rightarrow R$ satisfies the conditions of Remark 2 (if $n=2$ ) and Remark 3 (if $n \geq 3$ ).

Let $x \in I_{n 1}, y \in R$. Then we can write

$$
\begin{aligned}
\Delta\left(x y e_{n 1}\right) & =\Delta\left(x e_{n 1} y e_{11}+y e_{11} x e_{n 1}\right) \\
& =\Delta\left(x e_{n 1}\right) y e_{11}+x e_{n 1} \Delta\left(y e_{11}\right)+\Delta\left(y e_{11}\right) x e_{n 1}+y e_{11} \Delta\left(x e_{n 1}\right) .
\end{aligned}
$$

This implies

$$
e_{11} \Delta\left(x y e_{n 1}\right) e_{n n}=y e_{11} \Delta\left(x e_{n 1}\right) e_{n n} \text { i.e. } \quad f(x y) e_{1 n}=y f(x) e_{1 n} .
$$

Accordingly, $f(x y)=y f(x)$ for all $x \in I_{n 1}, y \in R$. Similarly, using $y x e_{n 1}=$ $y e_{n n} x e_{n 1}+x e_{n 1} y e_{n n}$ one shows that $f(y x)=f(x) y$ for all $x \in I_{n 1}, y \in R$. Therefore, $f\left(x^{2}\right)=x f(x)=f(x) x$ for all $x \in I_{n 1}$. Using also

$$
\begin{aligned}
0 & =e_{11} \Delta\left(\left(x e_{n 1}\right)^{2}\right)=e_{11}\left(\Delta\left(x e_{n 1}\right) x e_{n 1}+x e_{n 1} \Delta\left(x e_{n 1}\right)\right) \\
& =e_{11} \Delta\left(x e_{n 1}\right) e_{n n} x e_{n 1}=f(x) x e_{11},
\end{aligned}
$$

we see that $f\left(x^{2}\right)=0$ for all $x \in I_{n 1}$. Thus, in view of Remark $2, \delta$ is a singular Jordan derivation in case $n=2$.

Now let $n \geq 3$. Fix $j, 1<j<n$. Assume first that $x \in I_{n j}$ and $y \in I_{j 1}$. Then we can write

$$
\begin{aligned}
\Delta\left(x y e_{n 1}\right) & =\Delta\left(x e_{n j} y e_{j 1}+y e_{j 1} x e_{n j}\right) \\
& =\Delta\left(x e_{n j}\right) y e_{j 1}+x e_{n j} \Delta\left(y e_{j 1}\right)+\Delta\left(y e_{j 1}\right) x e_{n j}+y e_{j 1} \Delta\left(x e_{n j}\right),
\end{aligned}
$$


which yields $e_{11} \Delta\left(x y e_{n 1}\right) e_{n n}=f(x y) e_{1 n}=0$. Thus $f\left(I_{n 1} I_{j 1}\right)=0$. Next, let $x \in I_{n j}$ and $y \in I_{n 1}$. Since $x e_{n j} y e_{n 1}+y e_{n 1} x e_{n j}=0$, it follows that

$$
\Delta\left(x e_{n j}\right) y e_{n 1}+x e_{n j} \Delta\left(y e_{n 1}\right)+\Delta\left(y e_{n 1}\right) x e_{n j}+y e_{n 1} \Delta\left(x e_{n j}\right)=0 .
$$

Multiplying this identity from the left by $e_{11}$ and from the right by $e_{j j}$ we get

$$
0=e_{11} \Delta\left(y e_{n 1}\right) e_{n n} x e_{n j}=f(y) x e_{1 j}=f(x y) e_{1 j} .
$$

Therefore, $f\left(I_{n j} I_{n 1}\right)=0$. Similarly we see that $f\left(I_{n 1} I_{j 1}\right)=0$. Thus, by Remark $3, \delta$ is a singular Jordan derivation in case $n \geq 3$.

Note that $\Delta-\delta$ is a Jordan derivation satisfying:

$$
e_{11}(\Delta-\delta)\left(x e_{n 1}\right) e_{n n}=e_{11} \Delta\left(x e_{n 1}\right) e_{n n}-e_{11} \delta\left(x e_{n 1}\right) e_{n n}=0
$$

for all $x \in I_{n 1}$. Therefore, from now on we may assume without loss of generality that $\Delta: S \rightarrow S$ is a Jordan derivation with the property

$$
e_{11} \Delta\left(I_{n 1} e_{n 1}\right) e_{n n}=0 .
$$

Step 2. Let $1 \leq i \leq n$ and set $\Delta\left(e_{i i}\right)=\sum_{k, l} x_{k l}^{i} e_{k l}$. Since $e_{i i}$ is an idempotent, we have $\Delta\left(e_{i i}\right)=\Delta\left(e_{i i}\right) e_{i i}+e_{i i} \Delta\left(e_{i i}\right)$. Hence we see that $x_{i i}^{i}=0$ and $x_{k l}^{i}=0$ whenever both $k$ and $l$ are different from $i$. Thus we may write

$$
\Delta\left(e_{i i}\right)=\sum_{k \neq i} x_{k i}^{i} e_{k i}+\sum_{l \neq i} x_{i l}^{i} e_{i l} .
$$

Let $i \neq j$. As $e_{i i}$ and $e_{j j}$ are orthogonal idempotents, we have

$$
\Delta\left(e_{i i}\right) e_{j j}+e_{i i} \Delta\left(e_{j j}\right)+\Delta\left(e_{j j}\right) e_{i i}+e_{j j} \Delta\left(e_{i i}\right)=\Delta\left(e_{i i} e_{j j}+e_{j j} e_{i i}\right)=0 .
$$

Multiplying by $e_{i i}$ from the left and by $e_{j j}$ from the right we infer that $e_{i i} \Delta\left(e_{i i}\right) e_{j j}=$ $-e_{i i} \Delta\left(e_{j j}\right) e_{j j}$. That is,

$$
x_{i j}^{i}=-x_{i j}^{j}
$$

whenever $i \neq j$.

We now define the matrix $A=\sum_{i, j} a_{i j} e_{i j}, a_{i j} \in I_{i j}$ as follows. We set $a_{11}=0$ and define $a_{j j}$ according to

$$
a_{j j} e_{1 j}=-e_{11} \Delta\left(e_{1 j}\right) e_{j j} .
$$

Further, for $1 \leq i<j \leq n$ we set

$$
a_{i j}=-x_{i j}^{i}=x_{i j}^{j} .
$$


Thus, $a_{i j} e_{i j}=-e_{i i} \Delta\left(e_{i i}\right) e_{j j}=e_{i i} \Delta\left(e_{j j}\right) e_{j j}$. If $1 \leq j<i \leq n$, then we set

$$
a_{i j}=x_{i j}^{j}=-x_{i j}^{i},
$$

so that $a_{i j} e_{i j}=e_{i i} \Delta\left(e_{j j}\right) e_{j j}=-e_{i i} \Delta\left(e_{i i}\right) e_{j j}$.

Step 3. $d=\Delta-d_{A}$ is a Jordan derivation satisfying:

Lemma 2. $d\left(e_{i j}\right)=0$ for all $1 \leq i \leq j \leq n$.

Proof. Using (5), (8), and (9), for each $1 \leq i \leq n$ we have

$$
\begin{aligned}
d_{A}\left(e_{i i}\right) & =A e_{i i}-e_{i i} A \\
& =\sum_{k<i} a_{k i} e_{k i}+\sum_{i<k} a_{k i} e_{k i}-\sum_{l<i} a_{i l} e_{i l}-\sum_{i<l} a_{i l} e_{i l} \\
& =\sum_{k<i} x^{i}{ }_{k i} e_{k i}+\sum_{i<k} x^{i}{ }_{k i} e_{k i}+\sum_{l<i} x^{i}{ }_{i l} e_{i l}+\sum_{i<l} x_{i l}{ }_{i l} e_{i l} \\
& =\Delta\left(e_{i i}\right),
\end{aligned}
$$

so that $d\left(e_{i i}\right)=0,1 \leq i \leq n$. Now we compute $d\left(e_{1 j}\right)$ for each $j$ with $1<j \leq n$. Since $e_{1 j}=e_{11} e_{1 j} e_{j j}+e_{j j} e_{1 j} e_{11}$, it follows from (3) and $d\left(e_{11}\right)=d\left(e_{j j}\right)=0$ that

$$
d\left(e_{1 j}\right)=e_{11} d\left(e_{1 j}\right) e_{j j}+e_{j j} d\left(e_{1 j}\right) e_{11} .
$$

Applying $a_{11}=0$ and (7) we get

$$
\begin{aligned}
d\left(e_{1 j}\right)= & e_{11}\left(\Delta\left(e_{1 j}\right)-A e_{1 j}+e_{1 j} A\right) e_{j j} \\
& \left.+e_{j j}\left(\Delta\left(e_{1 j}\right)-A e_{1 j}+e_{1 j} A\right)\right) e_{11} \\
= & -a_{j j} e_{1 j}+a_{j j} e_{1 j}+e_{j j} \Delta\left(e_{1 j}\right) e_{11} \\
= & e_{j j} \Delta\left(e_{1 j}\right) e_{11} .
\end{aligned}
$$

Hence we see that $d\left(e_{1 j}\right)=b e_{j 1}$, for some $b \in I_{j 1}$. Using

$$
0=\Delta\left(e^{2}{ }_{1 j}\right) e_{11}=\left(e_{1 j} \Delta\left(e_{1 j}\right)+\Delta\left(e_{1 j}\right) e_{1 j}\right) e_{11}=e_{1 j} \Delta\left(e_{1 j}\right) e_{11},
$$

we therefore get $b e_{11}=e_{1 j} b e_{j 1}=e_{1 j} \Delta\left(e_{1 j}\right) e_{11}=0$. Thus $d\left(e_{1 j}\right)=0$.

Finally we compute $d\left(e_{i j}\right)$ for $1<i<j \leq n$. Since $e_{i j}=e_{i i} e_{i j} e_{j j}+e_{j j} e_{i j} e_{i i}$, we again have

$$
d\left(e_{i j}\right)=e_{i i} d\left(e_{i j}\right) e_{j j}+e_{j j} d\left(e_{i j}\right) e_{i i}
$$

Similarly as above we get

$$
d\left(e_{i j}\right)=e_{i i} \Delta\left(e_{i j}\right) e_{j j}-a_{i i} e_{i j}+a_{j j} e_{i j}+e_{j j} \Delta\left(e_{i j}\right) e_{i i} .
$$


Since $\Delta\left(e^{2}{ }_{i j}\right) e_{i i}=0$, it follows that $e_{j j} \Delta\left(e_{i j}\right) e_{i i}=0$. On the other hand, $e_{1 j}=$ $e_{1 i} e_{i j}+e_{i j} e_{1 i}$. Making use of $d\left(e_{1 j}\right)=d\left(e_{1 i}\right)=0$ it follows that $e_{1 i} d\left(e_{i j}\right)+$ $d\left(e_{i j}\right) e_{1 i}=0$. Hence

$$
\begin{aligned}
0 & =e_{1 i} d\left(e_{i j}\right) e_{j j}=e_{1 i}\left(\Delta\left(e_{i j}\right)-A e_{i j}+e_{i j} A\right) e_{j j} \\
& =e_{1 i}\left(e_{i i} \Delta\left(e_{i j}\right) e_{j j}-a_{i i} e_{i j}+a_{j j} e_{i j}\right),
\end{aligned}
$$

which gives $e_{i i} \Delta\left(e_{i j}\right) e_{j j}=\left(a_{i i}-a_{j j}\right) e_{i j}$ and $d\left(e_{i j}\right)=0$.

Define $\varphi: R \rightarrow R$ such that

$$
e_{11} d\left(x e_{11}\right) e_{11}=\varphi(x) e_{11} .
$$

Clearly $\varphi$ is an additive map.

Lemma 3. $d\left(x e_{i j}\right)=\varphi(x) e_{i j}$ for all $x \in I_{i j}$.

Proof. For any $1 \leq i \leq n$ we have $2 x e_{i i}=x e_{i i} e_{i i}+e_{i i} x e_{i i}$, so that

$$
d\left(x e_{i i}\right)=e_{i i} d\left(x e_{i i}\right) e_{i i} .
$$

Let $1<j \leq n$. Then we have

$$
\begin{aligned}
d\left(x e_{1 j}\right) & =d\left(x e_{11} e_{1 j}+e_{1 j} x e_{11}\right) \\
& =d\left(x e_{11}\right) e_{1 j}+e_{1 j} d\left(x e_{11}\right) \\
& =\varphi(x) e_{1 j} .
\end{aligned}
$$

On the other hand,

$$
\begin{aligned}
d\left(x e_{1 j}\right) & =d\left(x e_{j j} e_{1 j}+e_{1 j} x e_{j j}\right) \\
& =d\left(x e_{j j}\right) e_{1 j}+e_{1 j} d\left(x e_{j j}\right) \\
& =e_{1 j}\left(e_{j j} d\left(x e_{j j}\right) e_{j j}\right) .
\end{aligned}
$$

Therefore, $d\left(x e_{1 j}\right)=\varphi(x) e_{1 j}$ and $d\left(x e_{j j}\right)=\varphi(x) e_{j j}$ for every $x \in R$. Similarly we see that $d\left(x e_{i j}\right)=\varphi(x) e_{i j}$ whenever $1<i<j \leq n$.

Now let $1 \leq j<i \leq n$. Then

$$
\begin{aligned}
d\left(x e_{i j}\right) & =d\left(e_{i i} x e_{i j} e_{j j}+e_{j j} x e_{i j} e_{i i}\right) \\
& =e_{i i} d\left(x e_{i j}\right) e_{j j}+e_{j j} d\left(x e_{i j}\right) e_{i i}
\end{aligned}
$$

for all $x \in I_{i j}$. From $x e_{i j} e_{j i}+e_{j i} x e_{i j}=x e_{i i}+x e_{j j}$ one easily derives $e_{i i} d\left(x e_{i j}\right) e_{j j}=$ $\varphi(x) e_{i j}$ for all $x \in I_{i j}$. It remains to prove that $e_{j j} d\left(I_{i j} e_{i j}\right) e_{i i}=0$ whenever $1 \leq j<i \leq n$. First, using (4) we get

$$
\begin{aligned}
e_{11} d\left(x e_{n 1}\right) e_{n n} & =e_{11}\left(\Delta\left(x e_{n 1}\right)-A x e_{n 1}+x e_{n 1} A e_{11}\right) e_{n n} \\
& =e_{11} \Delta\left(x e_{n 1}\right) e_{n n}=0
\end{aligned}
$$


for all $x \in I_{n 1}$. Suppose $1<i<n$. Then $d\left(x e_{i 1} e_{i, i+1}+e_{i, i+1} x e_{i 1}\right)=0$, so that using $d\left(e_{i, i+1}\right)=0$ we get $e_{11} d\left(x e_{i 1}\right) e_{i i}=0$ for all $x \in I_{i 1}$. Finally, suppose $1<j<i \leq n$. Then

$$
0=d\left(x e_{i j} e_{1 j}+e_{1 j} x e_{i j}\right) e_{i i}=e_{1 j}\left(e_{j j} d\left(x e_{i j}\right) e_{i i}\right) .
$$

This means that $e_{j j} d\left(I_{i j} e_{i j}\right) e_{i i}=0$ and the lemma is proved.

Since for all $x, y \in R$ we have

$$
\begin{aligned}
\varphi(x y) e_{12} & =d\left(x y e_{12}\right)=d\left(x e_{11} y e_{12}+y e_{12} x e_{11}\right) \\
& =(\varphi(x) y+x \varphi(y)) e_{12},
\end{aligned}
$$

we see that $\varphi$ is a derivation on $R$. In view of Lemma 3 one observes that $d$ is a derivation arising from a derivation on $R$. Therefore, every Jordan derivation $\Delta$ : $S \rightarrow S$ can be written as the sum of a derivation $d: S \rightarrow S$ and a singular Jordan derivation $\delta: S \rightarrow S$. It remains to prove the uniqueness of this decomposition. Suppose $\Delta=d+\delta=d^{\prime}+\delta^{\prime}$, where $d, d^{\prime}$ are derivations and $\delta, \delta^{\prime}$ are singular Jordan derivations on $S$. Then

$$
\delta^{*}=d-d^{\prime}=\delta^{\prime}-\delta
$$

is a singular Jordan derivation such that

$$
\delta^{*}\left(x e_{n 1}\right)=f(x) e_{1 n} \text { and } \delta^{*}\left(x e_{i j}\right)=0 \text { for all } e_{i j} \neq e_{n 1},
$$

for some function $f: I_{n 1} \rightarrow R$. Since $\delta^{*}$ is also a derivation it follows that

$$
0=\delta^{*}\left(e_{11} x e_{n 1}\right)=e_{11} \delta^{*}\left(x e_{n 1}\right)=f(x) e_{1 n}
$$

for every $x \in I_{n 1}$. This implies that $\delta^{*}=0$, proving the uniqueness. The proof of Theorem 1 is thereby complete.

Finally we point out that if $d: S \rightarrow S$ is a derivation, then Theorem 1 immediately implies that $d$ is the sum of an inner derivation and a derivation arising from a derivation on $R$. Namely, as we saw, there do not exist nonzero singular Jordan derivations that are derivations. Also, the inspection of the proof shows that in this case we do not use the torsion assumption on $R$. Hence we get the first part of Corollary 1. For the second part we can use [2, Proposition].

\section{ACKNOWLEDGMENT}

I would like to thank the referee because of his most valuable suggestions, specially in the proof of Theorem 1. I wish also thank Professor G.H. Esslamzadeh because of his useful comments. 


\section{REFERENCES}

1. D. Benkovič, Jordan derivations and antiderivations on triangular matrices, Linear Algebra Appl., 397 (2005), 235-244.

2. S. P. Coelho and C. P. Milies, Derivations of upper triangular matrix rings, Linear Algebra Appl., 187 (1993), 263-267.

3. N. Jacobson, C. Rickart, Jordan homomorphisms of rings, Trans. Amer. Math. Soc., 69 (1950), 479-502.

4. S. Jøndrup, Automorphisms and derivations of upper triangular matrixrings, Linear Algebra Appl., 221 (1995), 205-218.

Nader M. Ghosseiri

Department of Mathematics,

Kurdistan University,

P.O. Box 416,

Sanandaj, Iran

E-mail: mnghosseiri@yahoo.com 